\title{
A Review on the Validity of Type-D Personality: From the Aspect of Construct and Predictability
}

\author{
Seung-Ah Jung1)
}

\begin{abstract}
Nearly 30 years after type-D personality was proposed as a psychological risk factor for cardiovascular disease, issues have been raised in the aspects of its validity. By reviewing the results of the existing major studies, this paper first outlined and summarized what the issues have been raised regarding the validity of type-D personality, and then proposed the future direction of research to resolve them. As a result of reviewing the main studies on the validity of the type-D personality, it can be concluded that the main issues raised so far converged in two aspects. One issue was about the validity of the psychological construct itself, whether the construct of type-D personality itself has its own sound ground. The other issue was whether the construct of type-D is really directly related to cardiovascular disease or whether it is an independent variable that can significantly predict the onset or prognosis of the disease. Although these two aspects of the issue exist, for now, the crucial rationale for the type-D personality to deny its validity appears to be weak. It is also expected that many of the current issues could be resolved if key factors in mediating(or moderating) between type-D personality and cardiovascular disease are identified in the future.
\end{abstract}

Keywords: Type-D Personality, Validity, Construct, Predictability, Cardiovascular Disease.

\section{The Background of Type-D Personality and Its Psychological Construct}

Since Freedman and Rosenman[1] proposed type-A personality as a personality risk factor for cardiovascular disease, we have an integrated view that the cause of medical disease are not confined to the domain of body. In additions, to fully understand this risk factor and its treatment, we must consider both body and mind in the process. Many follow-up studies have been conducted since this study, and alternative psychological risk factors have been explored because researchers failed to acquire consistent results with regards the relationship between type-A personality and cardiovascular disease. Efforts were made to explore the more stable personality construct that are more specifically associated with cardiovascular disease than other metabolic problems, while more precisely constructing the sub-factors of type-A. One of these $s$

Received(September 15, 2019), Review Result(1st: October 16, 2019, 2nd: November 21, 2019), Accepted(December 23, 2019)

1) (Professor) 61452 Dept. Counseling Psychology, Chosun Univ. 309 Pilmun-Daero, Dong-Gu, Gwangju, Korea

email: jsa@chosun.ac.kr 
was type-D personality, which was introduced by Denollet around the 1990s[2][3]. Even before he suggested type-D personality, it was known that psychological stress from negative emotions is associated with various medical problems. For example, studies have shown that it is not only a factor for the development of medical conditions such as hypertension or cardiovascular disease, but can also intensify depression and social isolation, which impedes the treatment of such medical conditions, worsens the prognosis and finally increases the mortality[4-7]. Denollet's stated that not only type-D has the tendency to suffer such negative emotions, but also other personality trait, which makes expressing them outwardly difficult and contributory to the development or prognosis of cardiovascular disease. In other words, he argued that people with both personality tendencies were vulnerable to cardiovascular disease. He defined these two dispositions as 'negative affectivity' (NA) and 'social inhibition' (SI) respectively and named 'type-D' personality as "Distressed" personality[3].

Of the two sub-constructs of the type-D personality he defined, NA refers to the tendency to experience overall negative emotions that causes discomfort and stress to an individual, including depression, anxiety, and anger, which was an important construct of type-A personality. NA is not a transient emotional state caused by specific external pressures, but a persistent tendency to experience such negative emotions across a variety of situations[8][9]. That is, people with NA personality are more susceptible and reactive to stress than others, and as a result, are more likely to experience and suffer from negative emotions. On the other hand, SI is an inhibitory trait that appears in social relations and is defined as the tendency to suppress emotions and behaviors in social interaction process', which is different from the existing concept of introversion. People with SI characteristics tend to be cautious. They overly control their emotions to avoid being negatively assessed in their interpersonal relationship with risks that might be dangerous. Thus, in interpersonal situations, they may seem reticent and atrophied.

One of the most frequently raised criticisms of Denollet's typ-D personality was that it seems to overlap many of the similar personality concepts already widely used. In other words, the construct of type-D personality is simply an "old wine in a new bottle."[10] However, criticism in this respect is unfair in light of Denollet's original definition of type-D personality and the background of his proposal. Type-D personality was not proposed by pure psychological interest, but it had a very special purpose to find the personality construct that cause exacerbation of chronic metabolic problems such as cardiovascular disease. In other words, the search for the personality trait of people with such diseases has shown that they experienced more often negative emotions, including depression, and at the same time was observed to 
have the tendency to fail expressing such negative emotions to others. Therefore, to be classified as a type-D personality as a psychological risk factor for cardiovascular disease, it is not enough to have only one trait of NA or SI.

With this exploration and discussion of personality constructs as a risk factor for cardiovascular disease, most researchers have focused on whether type-D personality really has a significant relationship with various aspects of cardiovascular disease. Many of these studies have supported Denollet's suggestion. However, it is necessary to review comprehensively what the main issues of these conflicting outcomes are and what should be further clarified in the future. Because many of the studies supporting Denollet's proposals and predictions were from Denollet himself or colleagues of his research group, researches have not always produced consistent results like many studies that has focused on the validity of type-A personality.

Hence, this paper intended to summarize the main issues concerning the validity of type-D personality in two aspects. First is the question of how valid and independent the psychological construct of type- $\mathrm{D}$ itself is, and the second is whether type-D personality is a construct that can predict the occurrence or worsening of cardiovascular disease in relation to the purpose for which the type-D personality was constructed. After a reviewing of these issues, this paper will suggests what future research is needed to resolve them.

\section{The Psychological Construct of Type-D Personality and Related Issues}

NA, defined as one of the two sub-constructs of type-D personality, 'which is the tendency to experience negative emotions,' already has similar psychological constructs. The most intensive issue has been neuroticism $(\mathrm{N})$, which is conceptualized as a psychological vulnerability that is susceptible to various neurotic disorders such as depressive and anxiety-related or somatized symptoms. Indeed, NA was consistently highly correlated with existing standardized measures of anxiety and depression[11-13]. SI is also related to various personality traits that have been conceptualized in relation to 'introversion'. For example, in Denollet's own study, measures of social interaction and performance anxiety, emotional or behavioral inhibition, and loneliness were classified as one factor. He also acknowledged that neurotic tendency correlated significantly with SI as well as NA, and confirmed SI also showed the most obvious negative correlation(-.52 -.65) with the 'extraversion' factor in such scales as NEO-FFI (NEO Five Factor Inventory) that is based on the theory of big-5 personality model and Eysenk personality scale(EPQ)[13][14]. From a conceptual and logical point of view, NA and SI do not necessarily have to be related given that a people who frequently experience negative emotions do not 
necessarily have to contract or be isolated socially. However, it has been reported that there is a moderate correlation between the two sub-constructs, and that the interaction model of the regression analysis also showed a significant interaction effect[14]. This was also related to the fact that the harm avoidance scale (HA) of trait and characteristic inventory (TCI-RS) was strongly correlated with both NA and SI. These results suggested the possibility that NA and SI may be a sub-construct of higher fundamental personality factors such as harm avoidance or neuroticism or can be integrated by those higher order personality constructs. These findings, are summarized in [Table 1].

[Table 1] Related Existing Constructs with NA and SI

\begin{tabular}{c|c|c}
\hline & Definition & Existing scales highly correlated \\
\hline \multirow{3}{*}{ NA } & $\begin{array}{c}\text { tendency or susceptibility to } \\
\text { experience negative emotions }\end{array}$ & $\begin{array}{c}\text { depression (BDI,D:MMPI-2), trait anxiety (STAI, Pt:MMPI-2), } \\
\text { negative emotionality (NEGE; MMPI-2), neurotic tendency } \\
\text { (N: NEO-PI-R), harm avoidance (HA: TCI-RS). }\end{array}$ \\
\hline SI & $\begin{array}{c}\text { tendency to inhibit emotion } \\
\text { and behavior in social } \\
\text { interaction situations }\end{array}$ & $\begin{array}{c}\text { social introversion (Si: MMPI-2), introversion (INT: MMPI-2, } \\
\text { E: NEO-PI-R, E-I:MBTI), harm avoidance (HA: TCI-RS), } \\
\text { social anxiety (SIAS, SPS) }\end{array}$ \\
\hline
\end{tabular}

NA: negative affectivity, SI:social Inhibition, BDI:Beck Depression Inventory, D: Depression scale, MMPI-2: Minnesota Multiphasic Personality Inventory, STAI:State-Trait Anxiety Inventory, Pt: Psychasthenia Scale, NEGE: Negative Emotionality, HA:Harm Avoidance, TCI-RS: Temperament and Character Inventory, Si: Social Introversion, SIAS:Social Interaction Anxiety Scale, SPS:Social Phobia Scale

In response to criticism about how SI differs from existing concept of introversion or repressive copying style, Denollet stated that while people who use defense mechanisms such as repression or denial reported less subjective pain or stress in the self-reporting test, people with high SI actually complain pain and do not showed defensive tendency[9][15]. After examining the independence of the two construct between type- $\mathrm{D}$ tendency and neurotic tendency of alexithymia which is closely related to defensive copying style of repression, Denollet concluded that the two personality construct were empirically separate psychological constructs[16-18].

On the other hand, against the criticism that the negative emotions included NA are largely overlapped with the depressive emotions, Pedersen and Denollet[19] found that the construct of NA differs from depression. In other words, in terms of the content of emotions, although NA, which is a sub-construct of type-D personality, is mainly related to internalized emotions like depression and anxiety. NA also includes externalized emotions such as anger and irritation. 
The most important difference is that NA is not a situational emotion (depression or anger) itself experienced in a particular situation, but a trait or persistent tendency to experience such negative emotions repeatedly across various situations. In addition, there is another important difference that people with depression do not necessarily suppress emotions in interpersonal relationship. While the people with type-D tendency, by definition, includes another inhibitory tendency called SI, both repeatedly experience negative emotions and at the same time inhibit those emotions. Indeed, with 1,012 healthy young people recruited from United Kingdom and Ireland, Williams et al[20] analyzed the validity about the type-D personality measured by D14. They suggested from this analysis that the type-D personality is an independent construct by showing that it maintains a significant relationship withe health-related behavior or social support even after controlling neurotic tendencies.

\section{The Predictive Validity of Type-D Personality}

Although there exist issues relating to the construct itself as discussed earlier, type-D personality is proposed in a practical purpose in exploring what psychological factors affect the onset or exacerbation of certain medical disease. Therefore, whether the psychological construct is valid or not can be limited only in the discussion of the conceptual aspect. What is more important practically is whether people classified as type-D personality can objectively demonstrate that they are actually more soundly related to disease-related factors (particularly cardiovascular diseases) than people who tend to be high in other similar psychological constructs. To date, the results of major systematic reviews and meta-analytic studies conducted to review the predictive validity of the type-D personality have led to the conclusion that such predictive validity is largely present[21-23]. But even with these systematic review studies, critical consideration is needed in that those studies are eventually based on previously published studies because the main findings related to type-D personality were mainly the work performed by Denollet and his colleagues who proposed the construct.

Prospective studies which are results of long-term follow-up with large-scale subjects have reported that type-D personality is a psychological risk factor that significantly affects the prevalence or prognosis of cardiovascular disease. Earlier, Denollet et al.[24] observed 308 patients diagnosed with coronary heart disease(CHD) for 6-10 years and compared mortality rates between type-D and non-D at the time of death. They found significant differences $(27 \%$ vs $7 \%$, which were persisted even after controlling other medical variables. Similar results were repeated in subsequent large prospective studies[25-29]. A cross-cultural study of 6,222 
patients with heart-related disease collected from 22 European countries showed that although the type-D personality was not related to the severity of heart disease, it was related to hypertension, smoking, inactivity, and depression, which are considered risk factors for heart disease[30]. In addition to cardiovascular disease, the type-D personality was also associated with various factors that exacerbate disease in patients with non-vascular disease[31], including disease awareness and lifestyle of rectal cancer patient[32], sleep problems and mental and physical symptoms[33], chronic pain, asthma, sleep apnea, brain damage, vaginitis, dizziness, and melanoma.

Although comprehensive review and meta-analysis generally support the predictive validity of type-D personality, some studies have found no association between type-D personality and cardiovascular disease. Other studies point out common problems with previous findings that have supported the predictive validity of the type-D personality. For example, after measuring type-D tendency, depression level and BNT (B-type natriuretic peptide) in 706 patients admitted for heart failure, their 18-month observation of them showed no type-D or depression effect, and only BNT had significant prediction of mortality[34]. This result is likely to be due to a defect in the categorical judgement of type-D suggested by Denollet or statistical bias in previous studies. Other studies have shown that although type-D personality affects the exacerbation of neurotic disorders including sleep problems, type-D personality showed any significant relevance to the incidence of mortality rate or symptom of myocardial infarction itself, no matter which statistical method is applied by categorical or dimensional classification[35]. de Voogd et al.[36] formed a large-scale subject, including those who participated in Denollet's and his colleagues' studies, and re-analyzed the data of 192 of them who died, and found no association between type-D personality and mortality. The authors argued that the results of meta-analysis by Denollet's Tilburg University team may be a kind of statistical illusion that resulted from a mixture of studies that reported large effect on small samples and small or no effect on large samples.

\section{Conclusions and Suggestions}

While reviewing existing major studies on type-D personality, which has been suggested after type-A personality as a psychological risk factor for cardiovascular disease, this paper also discussed important issues to be resolved in future studies related to type-D personality through the critical studies that began to be presented intermittently around 2010.

First, it is necessary to think about why criticisms have been raised, even though many large 
scaled prospective studies have supported the validity and predictability of type-D personality. One of the reasons is that most of the major studies related to type-D personality were largely the result of energetic research by Denollet himself and his colleagues who initially proposed the psychological construct of type-D. Although the researchers in this group directly refuted these criticisms through subsequent studies, or flexibly modified their views, the findings that have been mainly produced in the same research group with the same academic interest and repeated studies on the same subject may hold an element of implicit bias, even if there were no serious problems in the methodology. Accepting the acerbic criticism by Ioannidis[37], who warned the "risk of scientific inbreeding" that is likely to occur in the field of psychological research, studies by heterogenous research groups applying different classification and statistical method to different groups may be required to compare the results.

Second, criticism of the validity of the constructs of type-D personality itself cannot be resolved solely by pure theoretical discussion or personality psychological approach, or by an analysis of the scale of DS14. This is because the constructs of type-D itself were shaped from a health psychological perspective. There are existing similar constructs corresponding to NA and SI, respectively, and widely used measures that have already been standardized and used to measure them where similar results may be obtained with those measures. Therefore, the criticism that construct of type-D personality is virtually nothing new, as if it were "an old wine in a new bottle"[38] seems to be a legitimate argument. However, it should not be overlooked that the type-D constructs were built on the empirical and logical ground that it could predict the onset or prognosis of cardiovascular disease when the two sub-constructs of NA and SI exist simultaneously. Given this, it should not also be overlooked that the validity of the measure (DS14) made by Denollet as a tool for screening the type-D personality should be discussed with greater weight in terms of screening and predicting, and that the primary purpose of developing this scale was screening and predicting. DS14 consisted of only 14 items and in order to be classified as type-D personality with this scale, it is necessary to simultaneously score 10 points or more on both NA and SI sub-scales. Although there has been criticism that dimensional classification rather than categorical classification reflected the construct of type-D more realistically, recent studies comparing and analyzing the results of two classification methods suggested that both classification methods are valid with the categorical method showing superior effect[14].

Considering the research that has been going on in these two lines(construct validity-prediction), as well as the contradictory results and criticisms, future studies related to type-D personality should be directed toward exploring the major variables involved between 
the personality (type-D) and medical disease. In other words, it is necessary to explore what is the most decisive parameter or modulator that promote or alleviate the relationship between type-D personality and medical conditions, including cardiovascular disease. Demographic variables such as age, gender, and dietary habit, psychological variables such as behavioral patterns and lifestyles, and biological factors such as temperament or genetic predisposition would have produced the controversial results between the two points, type D personality and cardiovascular disease. The reason why health psychological or integrative view of disease emerges is that it is difficult to explain the cause of the disease with only a specific medical cause. We have learned that a cardiovascular disease is the result of a interaction of various factors from the psychological, biological and social aspects. If future studies do not fully reflect this complexity, it is likely that future conclusions about the type-D personality will follow similar steps as the study of the type-A personality.

\section{Acknowledgement}

This study was supported by research fund from Chosun University(2017)

\section{References}

[1] M. Friedman, R. H. Rosenman, Association of specific overt behavior pattern with blood and cardiovascular findings: blood cholesterol level, blood clotting time, incidence of arcus senilis, and clinical coronary artery disease, Journal of the American Medical Association, (1959), Vol.169, No.12, pp.1286-1296, DOI: 10.1001/jama.1959.03000290012005

[2] J. Denollet, Negative affectivity and repressive coping: pervasive influence on self-reported mood, health, and coronary-prone behavior, Psychosomatic Medicine, (1991), Vol.53, No.5, pp.538-556, DOI: 10.1097/00006842-199109000-00005

[3] J. Denollet, S. U. Sys, D. L. Brutsaert, Personality and mortality after myocardial infarction, Psychosomatic Medicine, (1995), Vol.57, No.6, pp.582-591, DOI: 10.1097/00006842-199511000-00011

[4] P. D. Somervell, B. H. Kaplan, G. Heiss, H. A. Tyroler, D. G. Kleinbaum, P. A. Obrist, Psychologic distress as a predictor of mortality, American Journal of Epidemiology, (1989), Vol.130, No.5, pp.1013-1023, DOI: $10.1093 /$ oxfordjournals.aje.a115402

[5] P. H. Silverstone, Depression and outcome in acute myocardial infarction, British Medical Journal, (1987), Vol.294, No.6566, pp.219-220, DOI: 10.1136/bmj.294.6566.219-a

[6] L. F. Berkman, L. Leo-Summers, R. I. Horwitz, Emotional support and survival after myocardial infarction: a prospective, population-based study of the elderly, Annals of Internal Medicine, (1992), Vol.117, No.12, pp.1003-1009, DOI: 10.7326/0003-4819-117-12-1003 
[7] R. B. Shekelle, S. W. Vernon, A. M. Ostfeld, Personality and coronary heart disease, Psychosomatic Medicine, (1991), Vol.53, No.2, pp.176-184, DOI: 10.1097/00006842-199103000-00007

[8] J. Denollet, Type D personality: A potential risk factor refined, Journal of Psychosomatic Research, (2000), Vol.49, No.4, pp.255-266, DOI: 10.1016/s0022-3999(00)00177-x

[9] J. Denollet, Personality and coronary heart disease: The Type-D Scale-16 (DS16), Annals of Behavioral Medicine, (1998), Vol.20, No.3, pp.209-215, DOI: 10.1007/BF02884962

[10] F. Lesperance, N. Frasure-Smith, Negative emotions and coronary heart disease: getting to the heart of the matter, The Lancet, (1996), Vol.347, No.8999, pp.414-415, DOI: 10.1016/s0140-6736(96)90004-5

[11] S. A. Jung, D. H. Oh, S. M. Lee, S. H. Kim, The Study about the Construct Validity of Type D Personality Scale : With Normal College Students Group as a Data Base, Journal of the Korean Neuropsychiatric Association, (2010), Vol.49, No.5, pp.628-633.

[12] S. Howard, B. M. Hughes, Construct, concurrent and discriminant validity of Type D personality in the general population: Associations with anxiety, depression, stress and cardiac output, Psychology \& Health (2012), Vol.27, No.2, pp.242-258, DOI: 10.1080/08870446.2011.603423.

[13] F. De Fruyt, J. Denollet, Type D Personality: A Five-Factor Model Perspective, Psychology and Health (2002), Vol.17, No.5, pp.671-683, DOI: https://doi.org/10.1080/08870440290025858

[14] I. Timmermans, H. Versteeg, S. Duijndam, C. Graafmans, P. Polak, J. Denollet, Social inhibition and emotional distress in patients with coronary artery disease: The Type D personality construct, Journal of Health Psychology, (2019), Vol.24, No.14, pp.1929-1944, DOI: 10.1177/1359105317709513

[15] N. Eisenberg, R. A. Fabes, B. C. Murphy, Relations of shyness and low sociability to regulation and emotionality, Journal of Personality and Social Psychology, (1995), Vol.68, No.3, pp.505-517, DOI: 10.1037/0022-3514.68.3.505

[16] M. A. Lumley, J. Tomakowsky, T. Torosian, The relationship of alexithymia to subjective and biomedical measures of disease, Psychosomatics, (1997), Vol.38, No.5, pp.497-502, DOI: https://doi.org/10.1016/S0033-3182(97)71427-0

[17] L. Williams, C. Curren, G. Bruce, Are alexithymia and Type D personality distinct or overlapping constructs? A confirmatory factor analysis of the Toronto alexithymia and Type D scales, Personality and Individual Differences, (2011), Vol.51, No.5, pp.683-686, DOI: 10.1016/j.paid.2011.06.007

[18] M. S. Epifanio, S. Ingoglia, P. Alfano, G. Lo Coco, S. La Grutta, Type D personality and alexithymia: common characteristics of two different constructs. Implications for research and clinical practice, Frontiers in Psychology, (2018). Vol.9, No.106, pp.1-9, DOI: 10.3389/fpsyg.2018.00106

[19] S. S. Pedersen, J. Denollet, Validity of the Type D personality construct in Danish post-MI patients and healthy controls, Journal of Psychosomatic Research, (2004), Vol.57, No.3, pp.265-272, DOI: 10.1016/S0022-3999(03)00614-7

[20] L. Williams, R. C. O'Connor, S. Howard, B. M. Hughes, D. W. Johnston, J. L. Hay, D. B. O'Connor, C. A. Lewis, E. Ferguson, N. Sheehy, M. A. Grealy, R. E. O'Carroll, Type-D personality mechanisms of effect: the role of health-related behavior and social support, Journal of Psychosomatic Research, (2008), Vol.64, No.1, pp.63-69, DOI: 10.1016/j.jpsychores.2007.06.008 
[21] P. M. Mommersteeg, F. Pouwer, Personality as a risk factor for the metabolic syndrome: a systematic review, Journal of Psychosomatic Research, (2012), Vol.73, No.5, pp.326-333, DOI: 10.1016/j.jpsychores.2012.08.019

[22] K. R. O'Dell, K. S. Masters, G. I. Spielmans, S. A. Maisto, Does type-D personality predict outcomes among patients with cardiovascular disease? A meta-analytic review, Journal of Psychosomatic Research, (2011), Vol.71, No.4, pp.199-206, DOI: 10.1016/j.jpsychores.2011.01.009

[23] G. Grande, M. Romppel, J. Barth, Association between type D personality and prognosis in patients with cardiovascular diseases: a systematic review and meta-analysis, Annals of Behavioral Medicine, (2012), Vol.43, No.3, pp.299-310, DOI: 10.1007/s12160-011-9339-0

[24] J. Denollet, H. Rombouts, T. C. Gillebert, D. L. Brutsaert, S. U. Sys, N. Stroobant, Personality as independent predictor of long-term mortality in patients with coronary heart disease, The Lancet, (1996), Vol.347, No.8999, pp.417-421, DOI: 10.1016/s0140-6736(96)90007-0

[25] E. J. Martens, N. Kupper, S. S. Pedersen, A. E. Aquarius, J. Denollet, Type-D personality is a stable taxonomy in post-MI patients over an 18-month period, Journal of Psychosomatic Research, (2007), Vol.63, No.5, pp.545-550, DOI: 10.1016/j.jpsychores.2007.06.005

[26] J. Denollet, S. S. Pedersen, Prognostic value of Type D personality compared with depressive symptoms, Archives of Internal Medicine, (2008), Vol.168, No.4, pp.431-432, DOI: 10.1001/archinternmed.2007.120

[27] A. A. Schiffer, O. R. Smith, S. S. Pedersen, J. W. Widdershoven, J. Denollet, Type D personality and cardiac mortality in patients with chronic heart failure, International Journal of Cardiology, (2010), Vol.142, No.3, pp.230-235, DOI: https://doi.org/10.1016/j.jicard.2008.12.090

[28] M. Romppel, C. Herrmann-Lingen, J. M. Vesper, G. Grande, Type D personality and persistence of depressive symptoms in a German cohort of cardiac patients, Journal of Affective Disorders, (2012), Vol.136, No.3, pp.1183-1187, DOI: 10.1016/j.jad.2011.11.044

[29] J. Denollet, S. S. Pedersen, C. J. Vrints, V. M. Conraads, Predictive value of social inhibition and negative affectivity for cardiovascular events and mortality in patients with coronary artery disease: the type D personality construct, Psychosomatic Medicine, (2013), Vol.75, No.9, pp.873-881, DOI: 10.1097/PSY.0000000000000001

[30] N. Kupper, S. S. Pedersen, S. Höfer, H. Saner, N. Oldridge, J. Denollet, J. Cross-cultural analysis of Type $\mathrm{D}$ (distressed) personality in 6222 patients with ischemic heart disease: A study from the International HeartQoL Project, International Journal of Cardiology, (2013), Vol.166, No.2, pp.327-333, DOI: 10.1016/j.ijcard.2011.10.084

[31] F. Mols, J. Denollet, A. A. Kaptein, P. H. Reemst, M. S. Thong, The association between Type D personality and illness perceptions in colorectal cancer survivors: a study from the population-based PROFILES registry, Journal of Psychosomatic Research, (2012), Vol.73. No.3, pp.232-239, DOI: 10.1016/j.jpsychores.2012.07.004

[32] F. Mols, J. Denollet, Type D personality among noncardiovascular patient populations: a systematic review, General hospital psychiatry, (2010), Vol.32, No.1, pp.66-72, DOI: https://doi.org/10.1016/j.genhosppsych.2009.09.010

[33] E. Condén, L. Ekselius, C. Åslund, Type D personality is associated with sleep problems in adolescents. Results from a population-based cohort study of Swedish adolescents, Journal of Psychosomatic Research, 
(2013), Vol.74, No.4, pp.290-295, DOI:10.1016/j.jpsychores.2012.11.011

[34] J. C. Coyne, T. Jaarsma, M. L. Luttik, E. van Sonderen, D. J. van Veldhuisen, R. Sanderman, Lack of prognostic value of type D personality for mortality in a large sample of heart failure patients, Psychosomatic medicine, (2011), Vol.73, No.7, pp.557-562, DOI: 10.1097/PSY.0b013e318227ac75

[35] E. Condén, Type D Personality: Psychometric Properties of the DS14 and Associations with Ill Health and Coronary Heart Disease in General and Clinical Populations, Acta Universitatis Upsaliensis, Doctoral dissertation, (2014)

[36] J. N. de Voogd, R. Sanderman, J. C. Coyne, A meta-analysis of spurious associations between type D personality and cardiovascular disease endpoints, Annals of Behavioral Medicine, (2012), Vol.44, No.1, pp. 136-137, DOI: https://doi.org/10.1007/s12160-012-9356-7

[37] J. P. Ioannidis, Scientific inbreeding and same-team replication: type D personality as an example, Journal of Psychosomatic Research, (2012), Vol.73, No.6, pp.408-410, DOI: 10.1016/j.jpsychores.2012.09.014

[38] J. Denollet, G. L. Van Heck, Psychological risk factors in heart disease, Journal of Psychosomatic Research, (2001), Vol.51, No.3, pp.465-468, DOI: 10.1016/S0022-3999(01)00230-6 